\title{
THE SURFACE EVOLUTION AND KINETIC ROUGHENING DURING HOMOEPITAXY OF GaAs (001)
}

\author{
B. G. OrR, M. D. Johnson, C. Orme, J. Sudijono and A. W. Hunt \\ The Harrison M. Randall Laboratory, University of Michigan, Ann Arbor, MI 48109-1120. U.S.A.
}

\begin{abstract}
Scanning tunneling microscopy studies have been performed on GaAs homoepitaxial films grown by molecular-beam epitaxy. Images show that in the earliest stages of deposition the morphology oscillates between one with two-dimensional islands and flat terraces. After the initial transient regime. the system evolves to a dynamical steady state. This state is characterized by a constant step density and as such the growth mode can be termed step flow. Comparison with reflection high-energy electron-diffraction (RHEED) shows that there is a direct correspondence between the surface step density and the RHEED specular intensity. Thick films (up to 1450 monolayers) display a slowly-increasing surface roughness. Analysis of the scaling properties and comparison with theories of film growth will be made.
\end{abstract}

Thin film deposition has become a critical technology for the advancement of modern electronics. A large number of artificial heterostructures have been produced by using various growth techniques. Molecular Beam Epitaxy (MBE) is particularly important because it affords monoatomic layer thickness control over films growing from the vapor phase at relatively low temperatures under supersaturation conditions[1]. This means that thermodynamically unstable structures can be made by tuning the growth rate and temperature. In fact, with careful choice of the growth parameters to control the surface kinetics, one can create different multi-layered structures in which individual layers maintain their chemical integrity and form compositionally abrupt interfaces with one another.

To understand the growth kinetics, one needs to focus on the growth process at the atomic level. The importance is clear. Semiconductor heterostructures and superlattices possess novel electrical and optical properties. For optimum performance of devices based on these artificial materials, the morphological sharpness of the interfaces is required. Roughness leads to increased carrier scattering in active regions, lowering the carrier mobility. A detailed microscopic examination of the surface kinetic processes will therefore enhance our ability to produce higher quality device structures. In this paper we concentrate on three major processes during MBE growth of GaAs on GaAs (001), i.e. nucleation, growth, and coarsening. Nucleation occurs when adsorbed adatoms make a random walk on the surface until they meet another adatom and form islands. Growth follows when islands larger than a critical nucleus extend with further attachment of adatoms. Coarsening is considered as the transition stage when growth approaches steady state. This occurs when the step density becomes so high that the growth mode evolves to step flow.
For many years, reflection high-energy electrondiffraction (RHEED) has been the standard in situ characterization tool for thin film MBE[2]. In addition to providing information on the evolution of surface structures, RHEED specular intensity oscillations are widely used to monitor growth from the transient (two-dimensional nucleation) regime to steady-state (step-flow) mode. However, being a macroscopic averaging diffraction technique, RHEED fails to give local topographic information. Efforts aimed at modeling nonequilibrium film growth phenomena are thus hindered due to the lack of a real space picture of the growth front as it evolves. This provides motivation for using scanning tunneling microscope (STM) to obtain real-space pictures of the surface evolution during growth.

The first part of these experimental studies is to examine the relationship between the RHEED oscillations and the surface evolution of GaAs during deposition. The second part has to do with the investigation of surface roughening during the steady-state growth, i.e. regime where RHEED specular beam no longer oscillates. The experiment consists of initiating growth from a recovered surface and then terminating deposition at a specific point during the growth process. This procedure is then repeated for various termination points. Because the samples are removed from the STM for regrowth, no direct comparison can be made between any specific feature in the progression of the images. We have imaged large areas at multiple sites on multiple samples. The images shown are thus representative of the surface.

Deposition was performed in a standard ultra-high vacuum system, base pressure $7 \times 10^{-11} \mathrm{~T}$. Effusion cells were used to produce both the $\mathrm{Ga}$ and $\mathrm{As}_{4}$ fluxes. Commercial GaAs (001) substrates were first chemically cleaned then loaded in the vacuum system where the oxide was removed at $580^{\circ} \mathrm{C}$ under an $\mathrm{As}_{4}$ flux. 
Prior to the experiments a $300 \mathrm{~nm}$ thick buffer layer was grown. The substrate temperature during deposition was $555 \mathrm{C}$. The As to $\mathrm{Ga}$ pressure ratio was 15 and the deposition rate was $0.18 \mu \mathrm{m} / \mathrm{h}$. The sample miscut as determined by STM was approx. $0.15 \mathrm{deg}$. The direction and magnitude of the local vicinality was found to vary appreciably. The incident angle of the RHEED beam was approx. $0.9 \mathrm{deg}$ and corresponded closely to the "in-phase" Bragg condition. The diffraction condition was chosen to yield a reduction in specularly scattered electron intensity as growth commences.

The key feature of the experiment was the ability to quench the surface morphology as it appeared during growth. To accomplish this a resistively heated low thermal-mass sample was used. The sample temperature could be reduced from that during growth $\left(555^{\circ} \mathrm{C}\right)$ to below $450^{\circ} \mathrm{C}$ in $1.5 \mathrm{~s} . \mathrm{A} \mathrm{LN}_{2}$ cooled baffle with a cooled shutter shrouds the sample[3]. The quench procedure has been described previously[4]. Upon the completion of quench, the sample was transferred in situ to the analysis chamber equipped with an STM. The RHEED intensity was recorded up to the time of the transfer. We found that the RHEED intensity is quite steady during this period indicating that no significant surface evolution has occurred.

Figure 1(a). shows an STM image of a recovered surface. The terrace size is large and the step edges are smooth. This is the GaAs surface as it appears before growth. Figure 1(b) shows the surface after deposition of 0.25 monolayer of GaAs. The typical size of
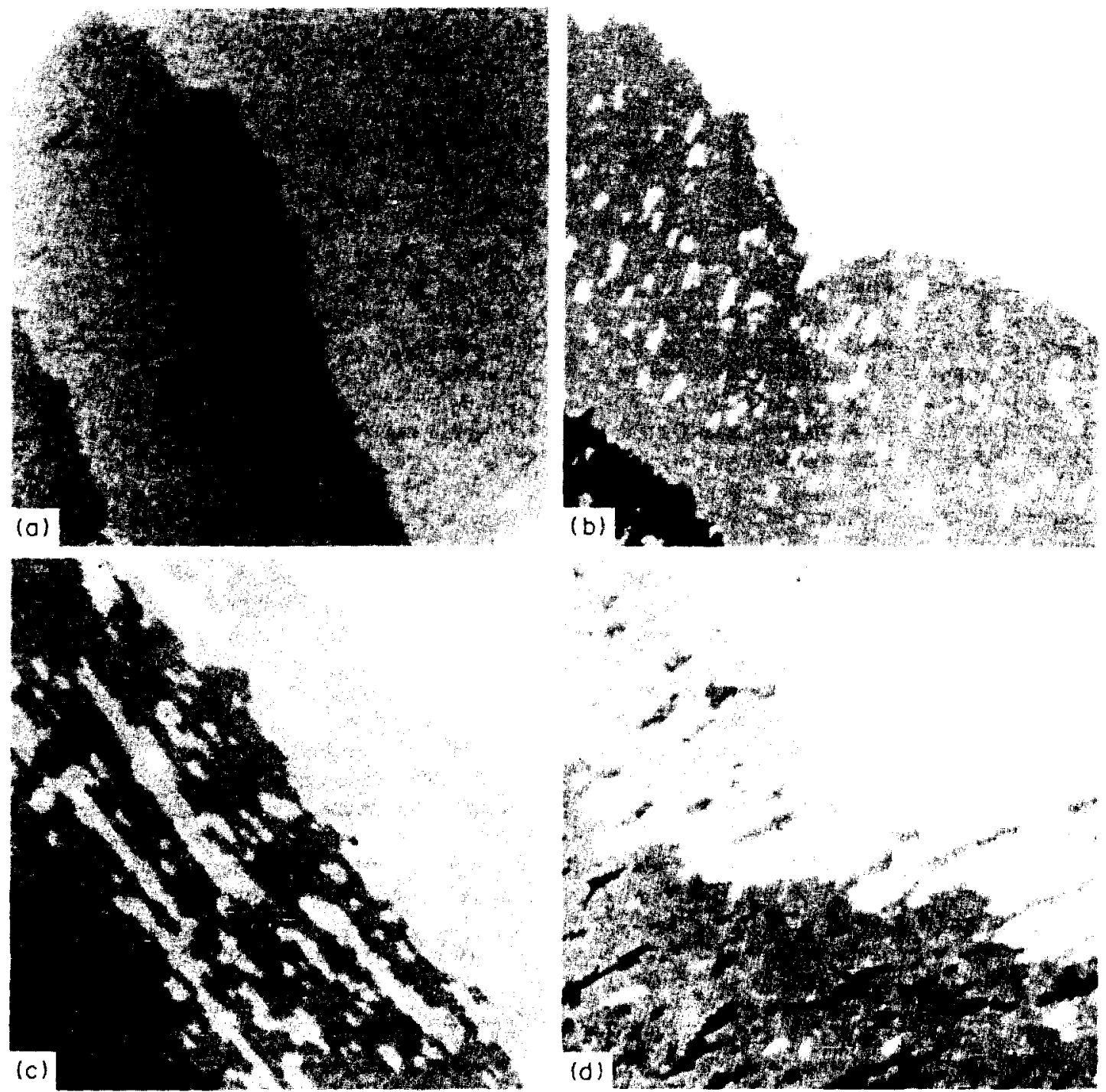

Fig. l. (a) STM image of a GaAs (00t) buffer layer. The scan range is $200 \times 200 \mathrm{~nm}$. The tunneling voltage $(V)$ was $+2.8 \mathrm{~V}$. applied to the sample. and the tunneling current $\left(I_{4}\right)$ was $80 \mathrm{pA}$. (b) STM image of GaAs (001) surface after deposition of 0.25 monolayer of GaAs. (c.d) STM image of GaAs (001) surface after termination of growth at the fourth RHEED minimum and maximum. Notice the change in the local direction of the viscinality in the figures. 


\section{Rheed oscillation data}

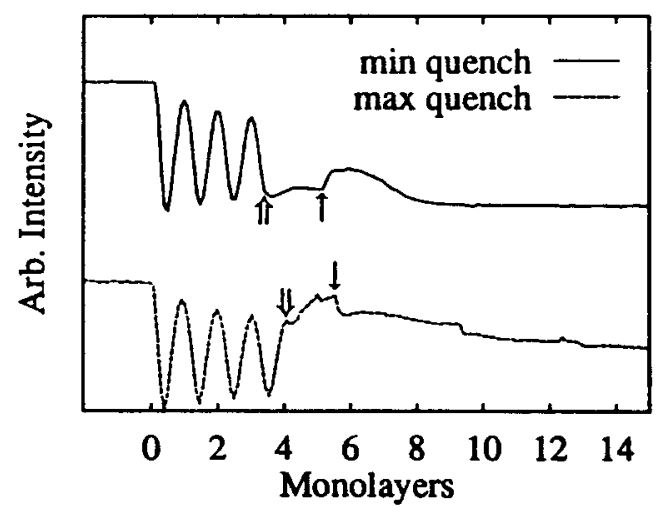

Fig. 2. RHEED specular intensity oscillations for GaAs on GaAs (001). The incident angle was $0.9 \mathrm{deg}$ and the azimuth was along [110]. The $\$$ labels the point at which growth was terminated for each sample and the $\downarrow$ indicates an experimental artifact due to the quenching procedure. STM data presented in (c) and (d) were acquired from these samples

islands and the average separation between islands are 80 and $300 \AA$ respectively. The coverage seems to be $<25 \%$ because growth may have occurred at some preexisting step-edges. Figure $1(\mathrm{c}, \mathrm{d})$ are respectively, images of the surface as it appeared at the fourth RHEED intensity minimum, and the fourth maximum during the oscillatory regime. The quenched RHEED curves are reproduced in Fig. 2 for completeness.

There is clearly a morphological change in the sample surface profile as it evolves from a RHEED maximum to a RHEED minimum. The surface quenched at an intensity maximum shows few islands on terraces and an approximately equal number of monolayer deep holes. In contrast, the surface quenched at a RHEED minimum shows many twodimensional islands on the terraces. There is a much lower density of monolayer deep holes. The terrace edges for both samples are relatively smooth.

To understand the observed morphological evolution in the context of temporal variation of the RHEED specular intensity, we briefly review various models on the interpretation of the diffraction process. One simple approach uses a kinematical approximation to determine the interaction of the scattered electrons with the surface[5]. In this picture the measured intensity is due to the interference of the electrons scattered from different terraces on the surface. At the correct incident angle (off-Bragg condition) this leads to an oscillation of the specularly reflected intensity due to a changing terrace occupation during deposition. As each growing layer proceeds from zero coverage through half filling and finally to a complete layer the specular intensity cycles

fOur STM data are not meant to rule out other models. The experimentally chosen diffraction condition provides little information on the models. through one period. A second, largely phenomenological, model which attempts to incorporate diffuse scattering has been proposed to explain RHEED oscillations. In this model the relevant quantity is not the terrace occupation but the step edge length per unit area, termed step density[6]. Steps provide a mechanism for diffuse scattering of the electron beam. With an increase in step density the specularly reflected intensity decreases. As in the previous model, if the surface morphology cycles from islanded to flat then the RHEED intensity would vary accordingly. Monte-Carlo growth simulations have shown an excellent correspondence between the step density and experimental RHEED data taken on vicinal surfaces[7]. There has been criticism of these models due to the incomplete treatment of multiple scattering (MS). While it has been experimentally demonstrated that the behavior of the scattered electrons is a complicated function of both azimuthal and polar angles[8], results from the dynamical approach[9] remain impractical due to stepped-surface structure idealization. However, much progress has been made in understanding RHEED through the MS approach. Mitura and Maksym[10] have recently used MS to successfully study the RHEED Azimuthal Plots, i.e. the specular beam intensity under rotation about the perpendicular axis of the surface. Furthermore, it has been shown that for the out-ofphase diffraction condition, the MS results for specular intensity behavior agree with that of the kinematical approach[11].

Our STM data can be interpreted within the context of the step density model. ${ }^{\dagger}$ The diffraction conditions correspond to the "in phase" Bragg condition where electrons reflected from adjacent terraces constructively interfere. The specular intensity varies not because of interference but, because of diffuse scattering from step edges. There is a correspondence between films with higher step density and lower RHEED specular intensity. On closer inspection, we find that, within a modified Born approximation, the Bragg scattering from holes $<5 \mathrm{~nm}$ in diameter contribute much less to the diffuse scattering than do islands and terrace edges. As a first approximation, if the step density contribution from small holes is subtracted from the total than the agreement between this modified step density and RHEED intensity is quite strong, see Fig. 4. This connection has been discussed in detail in a previous publication[4].

To further investigate the growth process, we have also examined the sample surface after the decay of RHEED oscillations. Figure 3(a) shows the surface after 60 monolayers have been deposited and the RHEED oscillation amplitude has decayed to $<5 \%$ of its original value. The sample is quenched in the same manner as before. The typical feature size has increased and in contrast to the earlier data the two-dimensional islands and terraces edges are now quite ramified. Figure 3(b) displays the surface after 
deposition of 120 monolayers. The surface in Fig. 3(a) has coarsened so much that it starts to roughen and nucleate two-dimensional islands on top of the terraces. This may explain the increase in step density between 60-monolyaer deposited surface and that of 120 monolayers. Nevertheless, the overall morphology remains flat, with about four layers present. Surprisingly, further growth does not seem to increase the surface roughness, as evidenced by Fig. 3(c,d). These STM images obtained after depositing 540 and 1450 monolayers [Fig. 3(c,d) respectively] show essentially identical topography to that of the 120 monolayer film. The rms roughness of these surfaces is $\sim 2.5 \AA$. This points to a central feature of the data, that is the decay of the oscillation amplitude has occurred without an increase in the interface roughness. As shown in Fig. 4(a), the interface width of the grown surface increases extremely slowly. Furthermore, the surface has achieve a steady state configuration, through a balance of island nucleation and step-edge attachment, which evolves with a constant step density. In short. the surface has reached the step flow growth mode.

During deposition an adatom can either diffuse to an existing upward step or kink on a terrace edge and be incorporated, diffuse to an existing downward step and be incorporated in the lower terrace, or collide with another adatom and form a new stable island. If the formation of new stable two-dimensional nuclei is dominant then the growth is called layer-bylayer[12]; however, if diffusion to an existing step dominates then the growth is termed step flow. The relative probability of an adatom to follow one of these trajectories is a complicated function of the substrate temperature, growth rate. and surface morphology. The experimental results indicate that

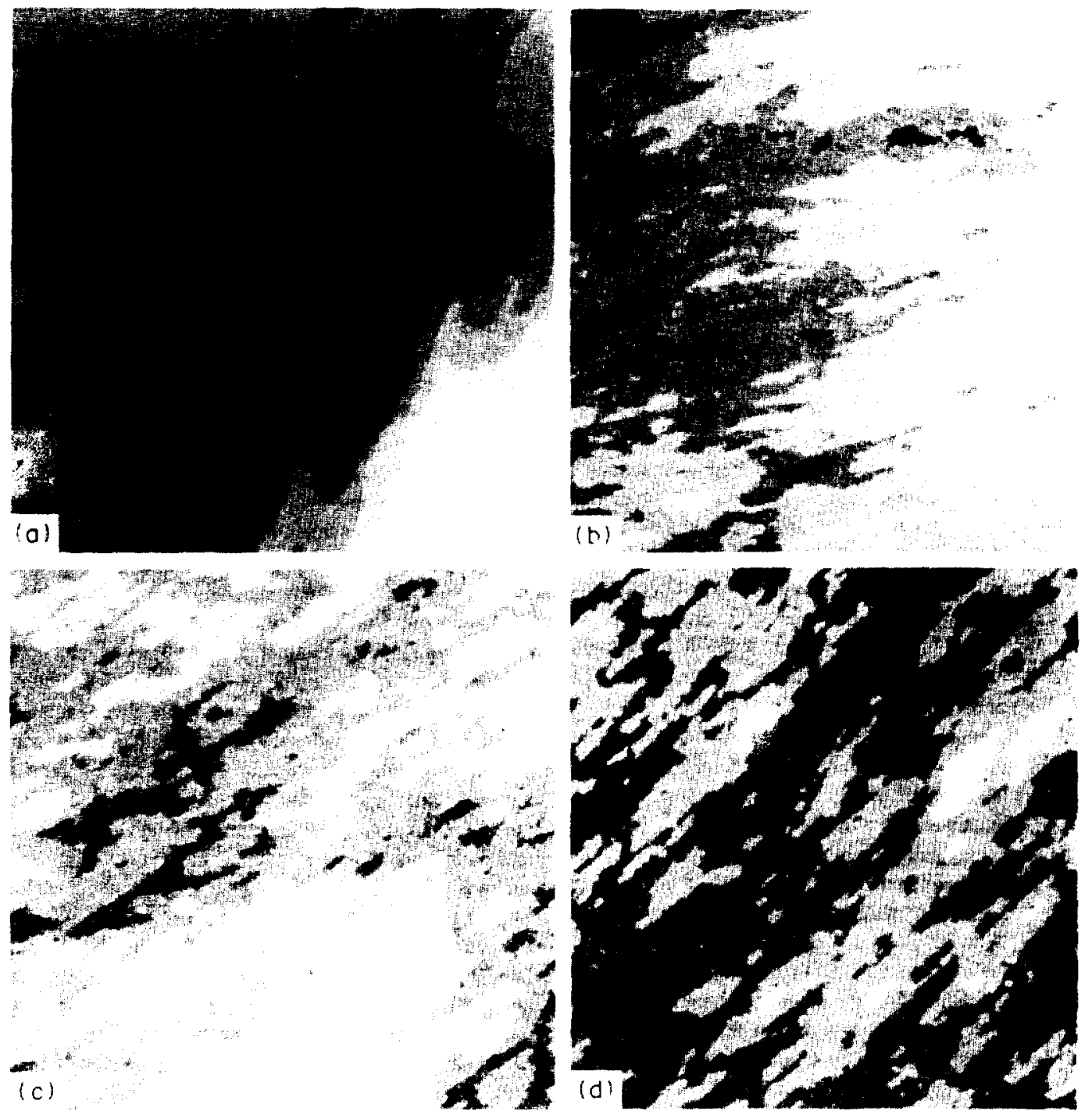

Fig. 3. (a) STM image of GaAs (001) after termination of growth of 60 monolayers (MLs). (b,c.d) STM images of GaAs (001) after deposition of $120 \mathrm{MLs}, 540 \mathrm{MLs}, 1450 \mathrm{MLs}$ respectively. The size of all images is $200 \times 200 \mathrm{~nm}$. 

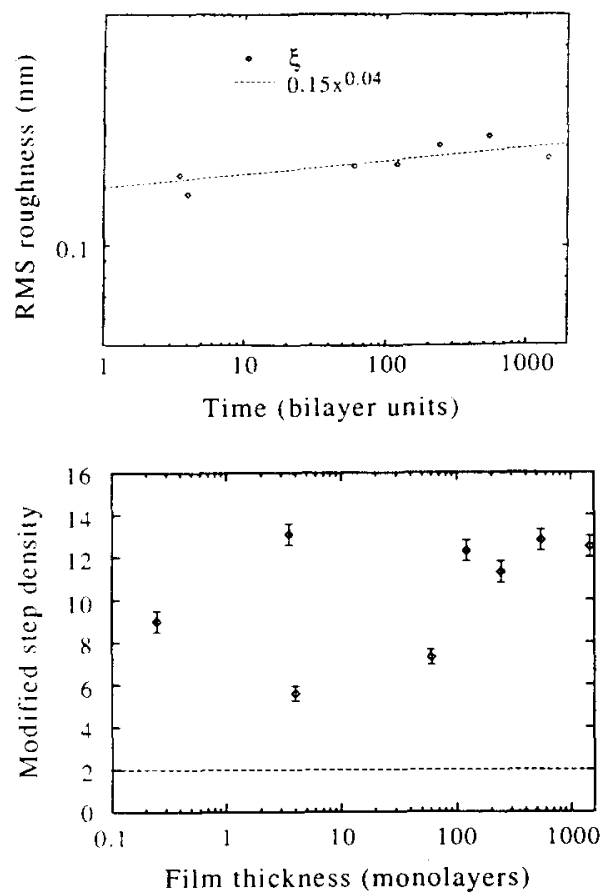

Fig, 4. (a) RMS roughness (in $\mathrm{nm}$ ) of the surfaces as a function of deposition time (bilayer units). The r.m.s. roughness is defined as $\sqrt{1 / N \Sigma\left(h_{i}-\hbar\right)^{2}}$ where $h_{i}$ is the height and the sum is over a $200 \times 200 \mathrm{~nm}$ area. (b) Plot of the surface step densities (in units of $10^{-2} \mathrm{~nm}^{-1}$ ) as a function of thicknesses (in monolayers). The modified step density is explained in the text. The dashed line represent the starting growth substrate.

evolution of growth of GaAs can be characterized by a change in the relative probability to nucleate a new island vs attaching to an existing stepedge. This evolution may be thought of as a dynamical transition to step flow growth. What is remarkable is that even though the substrate temperature and growth rate are constant, the surface morphology has been transformed to allow a new growth mode. This type of growth is not what is conventionally called step flow, i.e. a uniform progression of the steps across the surface. The classic step flow occurs when the substrate has a sufficient density of steps and very mobile adatoms. The new feature presented here is that deposition has dynamically generated a sufficient step density to allow for the change from layer-by-layer growth to step flow growth mode.

With this picture in mind, we will now perform a scaling analysis on the steady-state surface, i.e. during step-flow growth. Many theoretical models have been recently proposed to study kinetic roughening of surfaces grown with MBE[13-21]. The interest stems from the success at understanding such diverse growth phenomena as directed polymers in random media, evolution of bacterial colonies, UV Chemical

tThe conservative nature of $\mathrm{MBE}$ growth, i.e. no voids leads us to eliminate theories which do not conserve surface currents.
Vapor Deposition (UVCVD), and sedimentation in lake beds. These systems, it turns out, share common universality classes and display interesting non-trivial scaling behavior in the dynamics of their interfaces. However. there has not been agreement in how to apply these models to the case of MBE or in fact which universally class MBE growth falls under. This controversy is due in part to the lack of experimental evidence to decide which model and universality class is appropriate to describe MBE growth.

Despite the large amount of literature describing MBE growth, the only measurements of dynamic scaling in MBE growth roughening we know of are on films which were grown with low substrate temperatures and thus rough by MBE standards[22-24]. The diffusion length of an adatom on a terrace in these systems is relatively short and it is possible that these systems are governed by a different dynamics than that of typical MBE growth with rapid surface diffusion.

Dynamic renormalization group implies that in order to determine the asymptotic time evolution of a growing surface $h(\overline{\mathbf{x}}, t)$ one need only consider the contributions from long wavelength modes. The higher order terms are irrelevant and will not affect the long-time behavior. A universality class can be characterized by a simple equation for the growing interface involving only the relevant operators and from which the scaling properties can be determined. The primary quantities used to describe kinetic roughening are the asymptotic growth exponents $\alpha$ and $\beta$ which are expected to satisfy: $\xi^{2} \propto L^{2 x}$ $f\left(t / L^{x / \beta}\right)[25]$ where $\xi$ is the r.m.s. roughness, $L$ is the size of the system, and $f(x)=x^{2 \beta}$ for short times eventually saturating to a constant at $x \sim O(1)$.

Although $\mathrm{MBE}$ growth appears to be conceptually quite simple, i.e. no reevaporation of deposited particles and no formation of voidst an analytic description has not yet been determined to describe the scaling behavior. On the other hand, many numerical simulations of various growth models have been performed to determine the growth exponents $\alpha$ and $\beta$. Despite the many various models, a conclusive statement has yet to be made regarding $\mathrm{MBE}$ growth[14-20].

In an attempt to make comparison with the scaling theories of MBE growth we have examined the height-height correlation function defined as $C(x)=\left\langle(h(r)-h(r+x))^{2}\right\rangle r$. Figure 5 shows $C(x)$ for a surface after deposition of 1450 monolayers. The correlation function is predicted to scale as $C(x) \sim x^{2 x}[26]$. In Fig. 5 one can observe two distinct regimes in the correlation function. The small distance dependence indicates a maximum value for the growth exponent $\alpha \leqslant 0.2$. The extent over which this behavior is exhibited is approximately one decade in distance. For larger distances the behavior is dominated by the viscinality of the sample. For a miscut substrate (all physically realisable samples are miscut) asymptotically $C(x)$ approaches $x^{2}$. Our data shows 
this crossover at approximately $x=100 \mathrm{~nm}$. For our experiment the sample miscut was relatively small, $\sim 0.15$, producing a typical terrace width of $100 \mathrm{~nm}$. The STM images show quite clearly that during growth there is the dynamic generation of steps and roughness with lengths smaller than the terrace spacing. However, as shown in the dependence of the height-height correlation function $C(x)$ the longest wavelength behavior is dominated by the terrace width for deposition thicknesses up to 1450 layers. To determine the second growth exponent, $\beta$, the interface roughness as a function of time is needed. Referring to Fig. 4(a) it is seen that $\beta$, is approx. 0.04 . With these values of $\alpha$ and $\beta$ a comparison with the analytic models can be made.

Our measured exponents $x=0.2$ and $\beta=0.04$ show rather smooth growth and are in the best agreement with the $\mathrm{EW}$ values which predict logarithimic behavior. i.e. $\left(x=0^{+}, \beta=0^{+}\right)$. The discrepancy between the measured $x$ and the EW values is larger than that for measured $\beta$. This is most probably due to a greater effect which the vicinality has on surface correlations than on surface roughness.

We should mention at this point a need for caution when interpreting the experimental results in terms of theoretical models. To determine the growth exponents one needs to measure the roughness over a length scale $L$ which is sufficiently large to observe the continuum behavior of the system. At shorter lengths the surface morphology is determined by microscopic details which the scaling models cannot predict. With this caveat in mind we have performed atomic force microscopy (AFM) studies over very large areas of the samples. Figure 6 shows an image of a $\mathrm{GaAs}$ sample with $150 \mathrm{~nm}$ deposited. The surface is covered with large hills. The elongated mounds are $3 \mathrm{~nm}$ high and $0.4 \times 1.5 \mu \mathrm{m}$ in planar dimension. The slope of

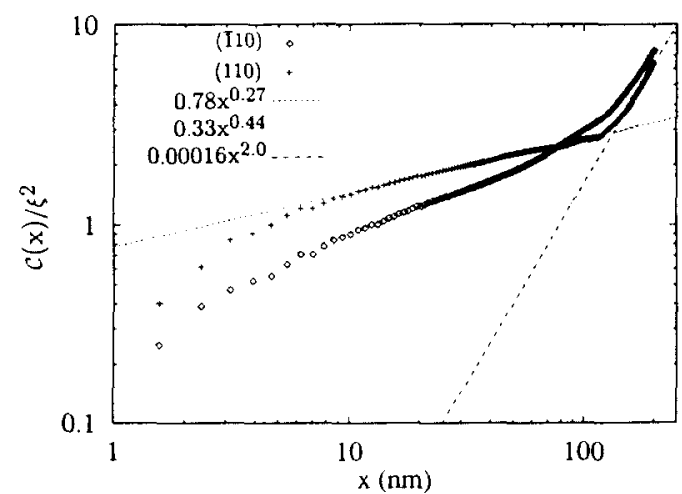

Fig. 5. A logarithmic plot. for two perpendicular crystallographic directions, of the normalized height-height correlation function, $C(x)=1 \xi^{2}\left\langle(h(r)-h(r+x))^{2}\right\rangle r$, where $\xi$ is r.m.s. roughness. The plots were calculated by averaging the height-height correlation function of 10 independent $200 \times 200 \mathrm{~nm}$ images which were plane subtracted so that terraces had no slope. The steep region of the function indicates the cutoff of scaling due to vicinality.
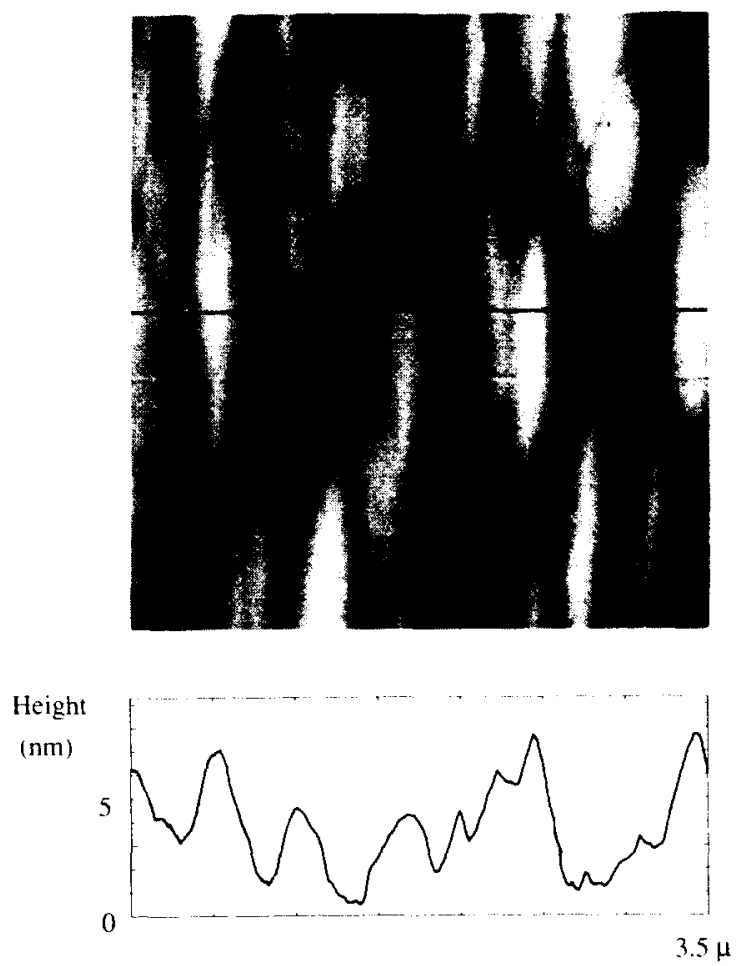

Fig. 6. AFM image of GaAs (001) surface after deposition of $150 \mathrm{~nm}$. The substrate temperature was 555 and the growth rate was 0.15 monolayer/sec. The grayscale range is $8 \mathrm{~nm}$ and the image scale is $3.5 \mu \mathrm{m}$ square.

the features is rather shallow, $\sim 1$, this corresponds to a terrace width of $18 \mathrm{~nm}$. Similar features have been observed by other groups[27].

Returning to the concept of a dynamical transition to step flow growth, one can now see that the steady-state surface morphology consists of regions on the surface where the terrace spacing is sufficiently small as to allow adatoms to attach to existing steps rather than nucleate new islands. One way in which a singular surface can achieve this state is to evolve to one with undulations. For the scaling theories of MBE growth the formation of these surface mounds is a critical test of the models. In fact. these structures indicate that the growth front is unstable and that none of the existing proposed scaling models for MBE growth is correct. There are however models which suggest that under certain conditions MBE growth is unstable $[19,28,29]$.

In conclusion, we have studied the evolution of the GaAs surface during MBE growth. Starting from a recovered substrate, upon the initiation of growth the surface progresses through a transient regime, where cyclic changes in the step density are found, to a steady state. Using in-phase diffraction conditions, we have shown a clear connection between the surface morphology in terms of step density and RHEED intensity oscillations. A notable result is that the decay of the RHEED oscillations is not due to an increase in surface width, but the dynamical 
evolution of the surface to step flow growth, defined as a local steady state with a constant step density. In this steady-state regime, the singular surface was found to be unstable and the gross surface morphology of the sample consisted of many mounds.

Acknowledgements - The authors would like to thank Dr D. Kessler. Professor L. M. Sander and Professor A. Zangwill for stimulating discussions. We also thank M. B. Elowitz for assistance with data analysis. This work has been supported by grants Nos N00014-89-J-1519 and NSF/DMR-8857828.

\section{REFERENCES}

1. B. A. Joyce, Contemp. Phys. 32, 21 (1991) and references therein.

2. P. K. Larsen and P. J. Dobson, Reflection High Energy Electron Diffraction and Reflection Electron Imaging of Surfaces, NATO ASI series B, Vol. 188. Plenum Press, New York (1988)

3. B. G. Orr, C. W. Snyder and M. D. Johnson, Rev. Sci. Instrum. 6, 1400 (1991)

4. J. Sudijono, M. D. Johnson, C. W. Snyder, M. B. Elowitz and B. G. Orr, Phys. Rev. Lett. 69, 2811 (1992).

5. P. R. Pukite, C. S. Lent and P. I. Cohen, Surf. Sci. 161, 39 (1985).

6. S. Clarke and D. D. Vvedensky, Phys. Rev. Lett. 58, 2235 (1987).

7. T. Shitara, D. D. Vvedensky, M. R. Wilby, J. Zhang, J. H. Neave and B. A. Joyce, Appl. Phys. Lett. 60, 1504 (1992).

8. P. J. Dobson, B. A. Joyce, J. H. Neave and J. Zhang, J. Cryst. Growth 81, 1 (1987)

9. T. Kawamura and P. A. Maksym. Surf. Sci. 161, 12 (1985).
10. Z. Mitura and P. A. Maksym, Phys. Rev. Lett. 70, 2904 (1993).

11. M. G. Knibb, to be published (1994).

12. J. H. Neave, B. A. Joyce. P. J. Dobson and N. Norton. Appl. Phys. A 31, 1 (1983).

13. S. F. Edwards and D. R. Wilkinson. Proc. R. Soc. Lond. A 381, 17 (1982)

14. M. Kardar, G. Parisi and Y. C. Zhang. Phys, Rer. Lett 56, 889 (1986).

15. D. E. Wolf and J. Villain, Europhys. Lett. 13, 389 (1990).

16. Z.-W. Lai and S. Das Sarma, Phys. Rev. Lett. 66, 2348 (1991).

17. S. Das Sarma and P. Tamborenea. Phys. Ret. Lett. 66 , 325 (1991).

18. D. A. Kessler, H. Levine and L. M. Sander, Phys. Ret. Lett. 69, 100 (1992).

19. J. Villain, J. Phys. I 1, 19 (1991).

20. H. Yan, Phys. Rev. Lett. 68, 3048 (1992).

21. M. Siegert and M. Plischke, Phys. Rer. Lett. 68, 2035 (1992).

22. J. Chevrier, V. Le Thanh, R. Buys and J. Derrien, Europhys. Lett. 16, 737 (1991)

23. Y.-L. He, H.-N. Yang, T.-M. Lu and G.-C. Wang. Phys. Rev. Lett. 69, 3770 (1992)

24. R. Chiarello, V. Panella, J. Krim and C. Thompson, Phys. Rev. Lett. 67, 3408 (1991).

25. F. Family, J. Phys. A: Math. Gen. 19, L441 (1986)

26. B. B. Mandelbrot, The Fractal Geometry of Nature. Freeman. New York (1982)

27. G. W. Smith, A. Pidduck, C. R. Whitehouse, J. L. Glasper and J. Spowart, J. Cryst. Growth 127, 966 (1993); T. Tiedje, private communication.

28. J. Krug, M. Plischke and M. Siegert. Phys. Rev. Lett. 70, 3271 (1993)

29. M. D. Johnson, C. Orme, A. W. Hunt, D. Graff, J. Sudijono, L. M. Sander and B. G. Orr, to be published. 